\title{
Detection and inversion of diggings water- bearing structure by using electrical imaging
}

\author{
Fengshan Ma, Qingyun Di, Kepeng Li \\ Key Laboratory of Shale Gas and GeoengineeringInstitute \\ of Geology and Geophysics,CAS \\ Beijing, China
}

\author{
Shanfei Wang, Wei Li \\ Shandong Gold Mining Company Limited, \\ Laizhou,Shandong, China
}

\begin{abstract}
The water-riched overburden of a seabed mine poses threat to safe exploitation. The level $-135 \mathrm{~m}$ strike drift of the Xinli mine district of Sanshandao gold mine in Shandong province was selected to be the survey site for electrical imaging, in order to map the groundwater distribution of the overlying rock under the level $-135 \mathrm{~m}$
\end{abstract}

Four methods, which are; Wenner, Dipole-Dipole, Schlumberger and P-S were used at the selected drift, respectively. The apparent resistivity and inversion results obtained from using the Wenner, Dpole-Dpole and Schlumberger array, gave consistent information about the subsurface medium, which is that three different resistive layers exists beneath the $-\mathbf{1 3 5} \mathrm{m}$ level. The first rock mass which is highly resistive has an average thickness of $\mathbf{3 0} \mathrm{m}$ and is located directly under the level $-135 \mathrm{~m}$, This indicates that little or no water exists in the rock mass between the levels of $\mathbf{1 3 5} \mathbf{m}$ and $\mathbf{- 1 6 5} \mathbf{m}$ since most of it have been discharged into $165 \mathrm{~m}$ drift. The rock mass ranging from $30 \mathrm{~m}$ to $60 \mathrm{~m}$ in depth under the $\mathbf{- 1 3 5} \mathbf{m}$ level has low resistivity and fracture water. The polarizability of the P-S method relates to metal sulfide. P-S inversion results show two local anomalies that occurred at $370 \mathrm{~m}$ and $440 \mathrm{~m}$ along the survey line at shallow depth between $10 \mathrm{~m}$ and $15 \mathrm{~m}$, which may be caused by rock mineralization. The obtained results show a good resemblance with the results obtained from hydrogeological surveys and hydrological structure analyses and that the gallery electrical imaging was able to map the water-bearing structures.

Keywords-detection; inversion; electrical imainging; waterbearing

\section{I.INTRODUCTION}

The high-resolution electrical method, which is also called resistivity tomography or electrical imaging method, was developed by in the 1980's. This method combines the abilities of both electric profiling and electrical sounding(Di et al.,1990), which can be used for engineering foundation and quality detection study, mining hydrogeological exploration, dam leakage detection and polymetallic orebody exploration in mining tunnel, etc ${ }^{[1]}$.

Electrical imaging method can automatically collect resistivity data, process the data on field, and monitor in real time the quality of the collected data. This method changes the traditional working style of the electrical resistivity method, reduces the labor intensity and improves data quality. Furthermore, the collection of large amount of data provides the information needed for precision data processing and interpretation, and electrical resistivity tomography. These greatly improves the electrical data processing and interpretation results and gives a reliable guaranty to exploration of small shallow target body.

The resistivity method is used to measure the electrical parameters of underground media by using electrodes laid on ground surface, wells and calculating the true resistivity, which is related to geological features such as metal, and properties such as rock-soil porosity and fluid saturation.

For this research, we selected $-135 \mathrm{~m}$ strike drift which is under seawater level from the Xinli mine district of Sanshandao gold mine in Shandong province, as a site for the application of electrical imaging method to detect groundwater distribution in rock mass below the sea level. This work will provide important geophysical data for seabed mining and hydrogeological study.

\section{II.METHODS AND PRINCIPLES}

The survey site was located in the level drift of Xinli gold mine $135 \mathrm{~m}$ below sea level. The drift was about $2 \mathrm{~m}$ wide and $2.5 \mathrm{~m}$ in height, where air was humid. Leaking seawater was seeping off walls and the drift floor was covered with mud and water. Though these conditions could make implementation difficult, electrodes were given favorable conditions in connecting them to the ground.

According to geophysical conditions, the working methods and projects of engineering geophysical exploration were formulated. One survey line was laid across the middle of the drift, about one meter away from each wall of the drift. The station spacing used was five meters and the data was acquired using 128 channels. The research was carried out along one survey line of length 635 $\mathrm{m}$ and employed four methods which are Wenner, DpoleDpole, Schlumberger and P-S method.

The electrical exploration system,E60BN, produced by Geopen which composes of host, switching cable, electrode bar and related software, was used in this study. The switching cable belonging to heptcable can connect multiple channels and is practically weightless. The host can automatically and conveniently generate constant voltage and current power supply. The grounded electrode bar was stainless steel electrode bar that is $40 \mathrm{~cm}$ in length and 6 $\mathrm{mm}$ in diameter. Because of low ground resistance, supply voltage was set to the constant value of $100 \mathrm{~V}$. Taking into 
consideration the working time and efficiency, the designed layer number was set to 42 layers for Wenner, 60 layers for Dipole-Dipole and 30 layers for Schlumberger and P-S (TABLE I).

\section{TABLE I. PARAMETER SETTINGS FOR DIFFERENT ELECTIRIC METHODS}

\begin{tabular}{|l|l|l|}
\hline & & \\
\hline method & $\begin{array}{l}\text { Line } \\
\text { length }\end{array}$ & \\
\hline wenner & & \\
\hline dipole & & \\
\hline schlumberger & & \\
\hline P-S & & \\
\hline
\end{tabular}

\begin{tabular}{|ccc|}
\hline Device and method & $\begin{array}{c}\text { Measuring line length } \\
(\mathrm{m})\end{array}$ & \begin{tabular}{c} 
Detecting layer number \\
\hline Wenner
\end{tabular} \\
\hline 635 & 42 \\
\hline Dpole-Dpole & 635 & 60 \\
\hline Schlumberger & 635 & 30 \\
\hline P-S & 635 & 30 \\
\hline
\end{tabular}

The resistivity method generally uses two current electrodes ( $\mathrm{C} 1$ and $\mathrm{C} 2)$ and two measuring electrodes (P1 and P2). Current (I) is passed into the subsurface and the potential difference between the two measuring electrodes is measured. Then, apparent resistivity can be calculated by using the following equation

$$
\rho_{s}=\frac{K \cdot V}{I}
$$

Where $\mathrm{K}$ is the coefficient related to the arrangement mode of the four electrodes.

The highly dense electrical method is a kind of array exploration method. The field measurement for this method will require the rearrangement of all the data from the electrodes (tens to hundreds) to their respective measured points. It takes the use of SPC electrode change-over switch and microcomputer engineering electric pilot to realize fast and automatic data collection. The microcomputer can process the measured data and give various physical interpretations in the form of geo-electric section.

Double-pole device, tri-electrode device and four-pole device are the three modes of observation of the highly dense electrical method. AMN and NMB of the tri-electrode method and symmetrical four-pole ,as well as dipole-dipole technique for the four-pole method are commonly used in practice. In other to acquire highly sufficient subsurface data and obtain better geological explanation, Wenner, DipoleDipole, Schlumberger and P-S methods were used. Their schematic diagrams are shown in Fig. 1, Fig. 2, Fig. 3 and Fig. 4, respectively.
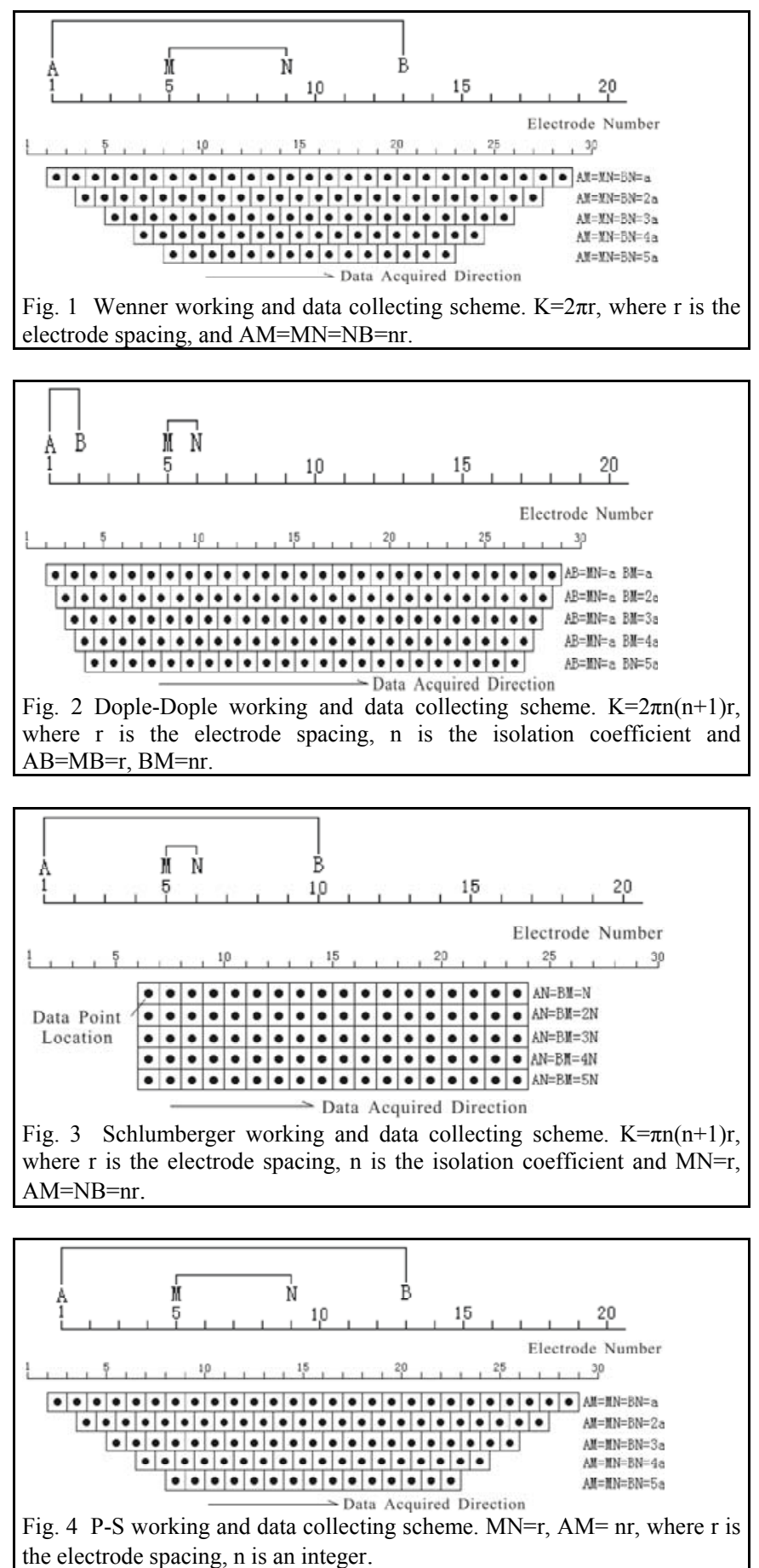

\section{III.CONCLUSIONS}

Highly dense electrical method was used to map the subsurface resistivity distribution over a $635 \mathrm{~m}$ long profile

- $\quad$ The inverse results obtained from using the Wenner, Dipole-Dipole and Schlumberger arrays were similar to each other and are characterized by three underground interfaces: The first rock mass which is highly resistive 
has an average thickness of $30 \mathrm{~m}$ and a high resistivity of about $600 \Omega \cdot \mathrm{m}$. This is followed by a conductive geoelectric layer with resistivity lower than $100 \Omega \cdot \mathrm{m}$, at depth ranging from $30 \mathrm{~m}$ to $60 \mathrm{~m}$. The last layer, at depth greater than $60 \mathrm{~m}$, is characterized by very high resistivity that is higher than $4000 \Omega \cdot \mathrm{m}$ (Fig. $5 \mathrm{~A}, 5 \mathrm{~B}$ and $5 \mathrm{C})$.

- The conclusion in (1) was obtained from the analysis of the horizontal layered medium at different depths. If horizontal analysis was done along the profiles, some subtle geological structures were found along the measuring line. The results from the Wenner array shows that the locations of $160 \mathrm{~m}$ depth related to the position $250 \mathrm{~m}---270 \mathrm{~m}$ have low resistivity. The Dipole-Dipole result reveals that the locations $160 \mathrm{~m}$, $240 \mathrm{~m}, 320 \mathrm{~m}, 400 \mathrm{~m}, 480 \mathrm{~m}$ and $530 \mathrm{~m}$ has low resistive body beneath them, of which $240 \mathrm{~m}, 320 \mathrm{~m}$ and $400 \mathrm{~m}$ are most obvious.

The parameter obtained from the dual frequency induced polarization method is the polarizability and it is directly related to the existence of metal sulfide. Its results show that there are two local anomalous bodies at shallow depth with value ranging from $10 \mathrm{~m}-15 \mathrm{~m}$, at distances $370 \mathrm{~m}$ and $440 \mathrm{~m}$ This may be caused by mineralization. It is also can be seen that the locations from $320 \mathrm{~m}$ to $400 \mathrm{~m}$ on measuring line in $60 \mathrm{~m}-80 \mathrm{~m}$ depth, display high polarizability which maybe reflects mineral veins (Fig.5D).

- $\quad$ The results obtained from this geophysical study are well consistent with the conclusions made from the hydrogeological surveys and hydrological structural analysis. In the vertical direction, $30 \mathrm{~m}$-thick rock show a high resistivity layer, which indicates that little or no water is present in the rock mass between the levels of $135 \mathrm{~m}$ and $-165 \mathrm{~m}$, where fractures have good connectivity and most of the previous water in them had been discharged into $-165 \mathrm{~m}$ drift through them. The location ranging from $30 \mathrm{~m}$ to $60 \mathrm{~m}$ depth has low resistivity, which means bears fracture water. And a highly resistive layer at depth greater than $60 \mathrm{~m}$, which reflects that the aquosity of the rock mass under $-200 \mathrm{~m}$ drift decreases progressively.

In the horizontal direction, low resistive anomalies were detected at locations $160 \mathrm{~m}, 183 \mathrm{~m}-243 \mathrm{~m}, 250 \mathrm{~m}-270 \mathrm{~m}$, $320 \mathrm{~m}, 400 \mathrm{~m}, 480 \mathrm{~m}$ and $530 \mathrm{~m}$, along the measurement line, which corresponds to the locations of water flowing fractures.

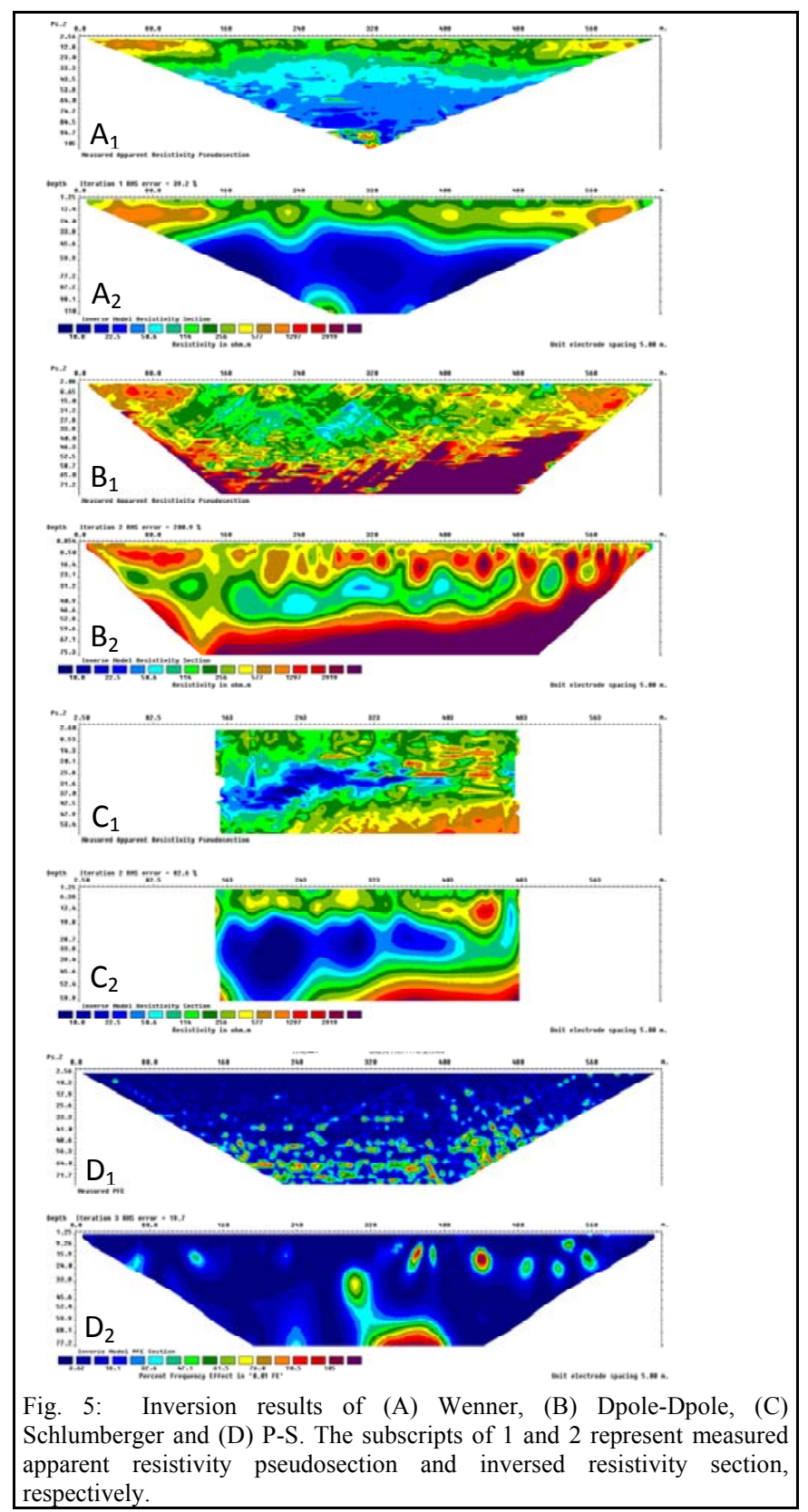

\section{REFERENCE}

[1] He, J. S., 1997, Development and prospect of electrical prospecting method: ACTC Geophysica Sinica, 40, 308-316. 